\title{
A Two-Stage Placement Algorithm with Multi-Objective Optimization and Group Decision Making
}

\author{
Daniela Borissova ${ }^{1,2}$, Ivan Mustakerov ${ }^{1}$ \\ ${ }^{1}$ Institute of Information and Communication Technologies, BAS, 1113 Sofia, Bulgaria \\ ${ }^{2}$ University of Library Studies and Information Technologies, 1784 Sofia, Bulgaria \\ Emails:dborissova@iit.bas.bg mustakerov@iit.bas.bg
}

\begin{abstract}
A two-stage placement algorithm with multi-objective optimization and group decision making is proposed. The first stage aims to determine a set of design alternatives for objects placement by multi-objective combinatorial optimization. The second stage relies on business intelligence via group decision-making based on solution of optimization task to make a choice of the most suitable alternative. The design alternatives are determined by means of weighted sum and lexicographic methods. The group decision making is used to evaluate determined design alternatives toward the design parameters. The described algorithm is used for wind farm layout optimization problem. The results of numerical testing demonstrate the applicability of the proposed algorithm.
\end{abstract}

Keywords: Placement algorithm, multi-objective alternatives determination, business intelligence, group decision making, wind farm layout design.

\section{Introduction}

The ultimate measure of decision quality expresses the degree to which the decision has led to the best business outcome. An advanced direction is using of business intelligence to access and analyse information to improve and optimize decisions and performance [21]. The complexity of decision making process is approached by technologies and best practices to deliver the knowledge to make the right decisions quickly and with confidence $[13,2]$. Business decisions are made on the base of the information for whole system incorporating financial and environmental aspects and rely on experts with capabilities to evaluate decision alternatives. The problem of determination of variety of alternatives for placement of objects and choosing the best one by group decision making arises in many practical problems. One such problem is designing of wind farm layout to maximize the energy output and considering wind conditions, technical requirements and other different restrictions [1]. The optimal placement of wind turbines that ensures maximum efficiency is essential in designing of wind farm project in both cases of onshore and offshore wind farm. Therefore, the proper optimization is imperative in designing renewable 
energy system for maximizing its cost effectiveness. Designing of renewable energy system considers the problem of placement of turbines with known dimensions in the farm area to minimize the cost and maximize the system efficiency. Many research articles are subject of Wind Farm Layout Optimization Problem (WFLOP) and different approaches and various optimization algorithms are proposed as: genetic algorithms [4, 15, 24, 26, 40], pattern search algorithm [7], evolutionary algorithm $[25,35,39]$. An alternative approach to WFLOP is using of the mathematical programming optimization methods $[3,5,6,19,27,34]$. Due to the complex and multi-disciplinary nature of WFLOP many conflicting objectives are to be involved during the design process. In this sense, multi-objective optimization modelling can more accurately reflect real life situations and are therefore more suitable tool to deal with WFLOP $[4,20,38]$. From the managerial point of view it is hard to take decision on the basis of single alternative. More substantiated decision would be reached if different design alternatives conforming to different design requirements are compared to get to the best design solution [19]. The design of wind farm is multidimensional process and involves different aspects - economic, technical, environmental, social, etc. This means that different design alternatives are to be defined and evaluated by a group of experts to make the most suitable business decision. Aggregating different experts' evaluations over set of alternatives to select a single collective alternative can be done by Group Decision Making (GDM) [12]. The GDM is based on evaluations of multiple alternatives against multiple criteria by variety of experts to provide better decisions [2]. In many cases different points of view about the criteria and their importance leads to conflicting evaluations of alternatives which complicates the GDM. The further complication arises when importance of the opinions of experts themselves are to be considered according their positions in the project management. When the number of the criteria and alternatives is finite, and the alternatives are given explicitly are known as Multi-Attribute Decision Making (MADM). The problems MADM can be tackled by different models and methods [29-33]. An important part of modern decision science is MultiAttribute Group Decision Making (MAGDM) where the process of decision is made by multiple decision makers as assessment of alternatives over a range of attributes. Two main directions in the MADM methods can be distinguished - methods based on the Multi-Attribute Utility Theory (MAUT) and outranking methods [12, 16]. In this article MAUT principles are realized by combinatorial optimization formulation that takes into account the different experts' opinions about the importance of criteria (wind farm parameters), DMs' evaluations of alternatives and weights of DMs.

The main contribution of the article proposes two-stage algorithm which combines multi-objective optimization as design simulation tool and GDM based on single-objective integer linear programming model, to get numerically reasoned optimal design alternative. The rest of the article is structured as follows: Section 2 describes in details the proposed two-stage algorithm, Section 3 describes the algorithm application for WFLOP; Section 4 illustrates the applicability of proposed approach by numerical testing. In Section 5 analysis of the numerical testing results and discussions are presented, and conclusions are given in Section 6. 


\section{A two-stage placement algorithm with multi-objective optimization and group decision making}

This section focuses on description of the proposed two-stage algorithm for determination of design alternatives and choice of most suitable alternative (Fig. 1).

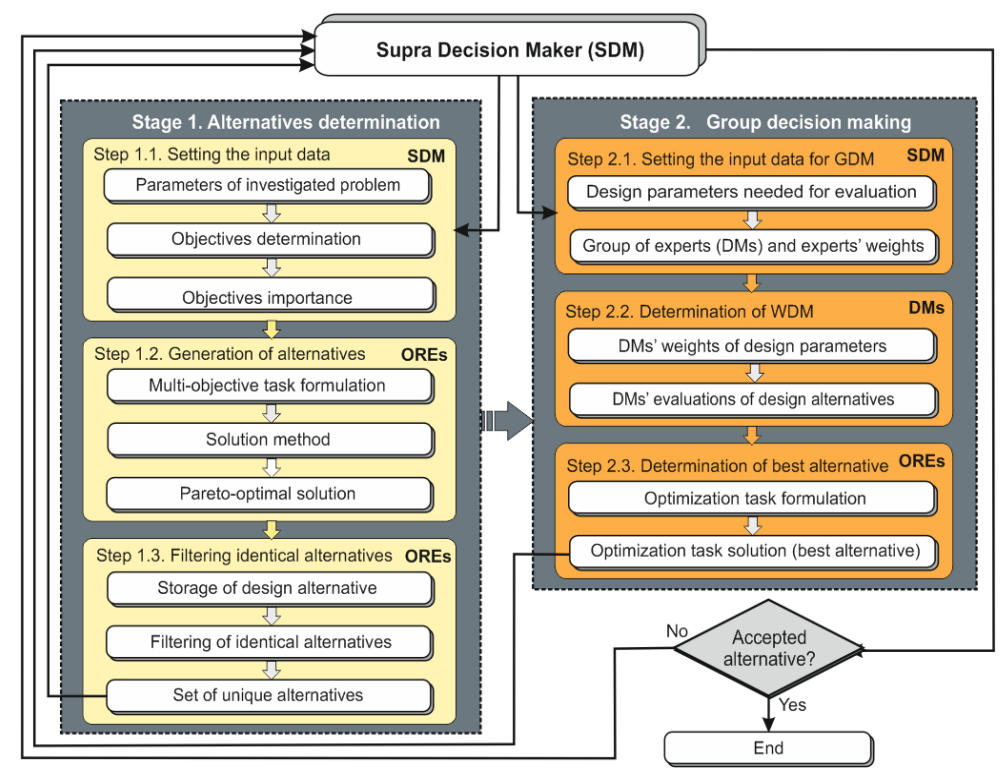

Fig. 1. Flowchart of the proposed two-stage algorithm

The two-stage algorithm for placement consists of: 1) determination of a set of design alternatives conforming to different design scenarios; 2) choice of the best alternative by GDM. It is assumed that overall project management is done by a Supra Decision Maker (SDM). The SDM is authorized to organize the overall decision making process, to gather a group of different experts (DMs) relevant to the project design aspects and to collect experts' evaluations of different alternatives. The SDM is usually a manager who is responsible to propose a final decision at a higher level of management or has the right to make the final decision by himself.

The responsibilities for input data about the parameters, objectives and their importance are assigned to SDM on the Step 1.1 (Fig. 1). The goal of multi-objective optimization problem is to identify Pareto-optimal solution using additional information from DM about the objectives importance. On the Step 1.2 the experts in Operation REsearch (OREs) have to formulate and to solve multi-objective optimization tasks. They choose and implements proper multi-objective solution method and express objectives importance. A common approach for dealing with multi-objective optimization problems is to use scalarization techniques $[8,14,23]$ due to their simplicity and effectiveness. As a result of multi-objective task solution, a Pareto-optimal design alternative is determined. Then, on Step 1.3, design alternatives are filtered to get a set of unique alternatives. 
The input data for GDM is implemented in Step 2.1. SDM determines the evaluation criteria and group of experts (DMs) from different knowledge domains and defines some weight coefficients for each of DMs. Weights of DMs play a very important role in MAGDM because they reflect the expertise of each particular expert. The range of weights for experts could be limited within interval of 0 to 1 , where 1 means the most experienced and trusted level of expertise [12].

The essence of Step 2.2 is determination of weighted decision matrix. A key point in decision making is determination of relevant evaluation criteria because they significantly influence on the selection process. Usually these criteria are given by high level management. Regardless of who sets the criteria and alternatives, these two parameters are mandatory elements in WDM.

On Step 2.3 a single criterion optimization modelling is used to formulate an optimization task for selection of the best alternative. This solution is presented to SDM for a decision - to accept the determined alternative or to repeat stages of algorithm.

\section{Application of two-stage algorithm for design of WFLOP}

Accordingly to the first stage of the described algorithm, the set of alternatives that define various wind farm layouts are generated by using of multi-objective optimization as follows:

Step 1.1. Collecting of the input data - set of different types of wind turbines and characteristics of wind site and wind conditions. SDM plays a crucial role in negotiating of the phrasing of the design objectives and in determination of their importance.

Step 1.2. The multi-objective combinatorial optimization is used as an analytical simulation tool for different wind farm layout design alternatives. For the goal a combinatorial multi-objective optimization model is proposed. It allows simultaneously determination of turbines' type and number, and their placement positions. The overall objective of a wind farm project accepted by many researchers is to increase the Annual Energy Production (AEP) while reducing the costs. These two objectives are used to simulate different wind farm layout design scenarios by multi-objective combinatorial optimization:

subject to

$$
\begin{gathered}
\max \mathrm{AEP}=h_{y} \eta N P_{\mathrm{wt}} \\
\min \text { Costs }=N\left(\frac{2}{3}+\frac{1}{3} e^{-0.00174 N^{2}}\right),
\end{gathered}
$$

$$
\begin{aligned}
& N=N_{x} N_{y}, \\
& N_{x}=\left(L_{x} / \mathrm{SD}_{x}\right)+1, \\
& N_{y}=\left(L_{y} / \mathrm{SD}_{y}\right)+1, \\
& \mathrm{SD}_{x}=k_{x} D_{\mathrm{wt}}, \\
& \mathrm{SD}_{y}=k_{y} D_{\mathrm{wt}}, \\
& k_{y}^{\min } \leq k_{y} \leq k_{y}^{\max }, k_{y}>0, \\
& k_{x}^{\min } \leq k_{x} \leq k_{x}^{\max }, k_{x}>0,
\end{aligned}
$$




$$
\begin{aligned}
P_{\mathrm{wt}} & =\sum_{i}^{m} x_{i} P_{\mathrm{wt}}^{i}, \\
D_{\mathrm{wt}} & =\sum_{i}^{m} x_{i} D_{\mathrm{wt}}^{i}, \\
\sum_{i}^{m} x_{i} & =1, x \in\{0,1\},
\end{aligned}
$$

where the AEP is represented by the number of hours over the year $\left(h_{y}\right)$, coefficient of the nominal wind power utilization $(\eta)$ [27], number of turbines $(N)$ and turbines' rated power $\left(P_{\mathrm{wt}}\right)$. The second objective is non-dimensional costs per year $N\left(\frac{2}{3}+\frac{1}{3} e^{-0.00174 N^{2}}\right)[15,24,36]$ expressed as a function of total turbines number $(N)$ while $N_{x}$ is the number of turbines in rows and $N_{y}$ is turbines number in columns. Rated power of $i$-th wind turbine is denoted by $P_{\mathrm{wt}}^{i}$ with corresponding rotor diameter $D_{\mathrm{wt}}^{i}$. The notations $L_{x}$ and $L_{y}$ are used for dimensions of wind farm area. The decision variables (unknowns) $x_{i} \in\{0,1\}$ are used in the model (1)-(11) to choose the type of turbines. Coefficients of turbines separation distances in rows and cols $k_{x}$ and $k_{y}$ are used to determine the turbines separation distances respectively $\mathrm{SD}_{x}$ and $\mathrm{SD}_{y}$. The negative influence of the WE is taken into account by introduction of separation coefficients $k_{x}$ and $k_{y}$ as variables. These coefficients are used to define the separation distances (as number of turbine's diameter) needed to avoid the influence of WE. The separation coefficients $k_{x}$ and $k_{y}$ are limited to some upper and lower boundaries accordingly the recommendations $[5,15,24,37]$ or can be calculated for particular wind farm site with given wind conditions.

It should be noted here that application of the proposed two-stage algorithm is not restricted to these two objectives and other formulations for the objectives can be used.

Step 1.3. On this step the determined alternative is stored for later comparison and assessment by group of DMs. The SDM contributes in simulation of other design scenarios by repeating the Step 1.1 with changed preferences about the objectives importance or by requesting for another multi-objective solution method. When different design scenarios are simulated, the determined alternatives are filtered, i.e., identical alternatives are considered as one unique alternative and all unique alternatives are stored for later evaluation on Stage 2.

Step 2.1. On this step the SDM determines the wind farm parameters as evaluation criteria and selects a group of experts that represent different design aspects of wind farm project - engineers, economists, ecologists, etc. SDM defines also DMs' weighted coefficients according their expertise toward the project goals.

Step 2.2. This step is based on MADM described by a Weighted Decision Matrix (WDM). This WDM indicates both the set of alternatives and the set of criteria being considered in a problem. The structure of WDM proposed in the article includes: DMs' weights for the importance of the design parameters (criteria); DMs' scores towards design alternatives' parameters; and weighted coefficients for DMs. Each of the DMs estimates alternatives independently, without negotiations with other DMs and accordingly to his specific point of view. For example, a financial expert probably will put more weight on financial parameters of the design while engineers would be more interested in wind farm energy production. The SDM assigns weighting coefficients for each DM according to their hierarchy positions in the management of WFLOP. 
In general case, the different DMs' points of view about alternatives can be conflicting because they reflect different preferences toward importance of design parameters. In WDM the higher values of evaluations mean a better performance and the final goal is to maximize the outcome of decision about the best alternative.

Step 2.3. On this step, single criterion optimization modelling is used to formulate an optimization task as: subject to

$$
\max \sum_{i=1}^{M} \sum_{k=1}^{K} \alpha^{k} w_{i}^{k} A_{i}^{k},
$$

$$
\begin{aligned}
& \forall i=1,2, \ldots, M:\left(\forall k=1,2, \ldots, K: A_{i}^{k}=\sum_{j=1}^{J} a_{i, j}^{k} x_{j}\right), \\
& \sum_{j=1}^{J} x_{j}=1, x_{j} \in\{0,1\}, \\
& \alpha^{k} \in(0,1),
\end{aligned}
$$

where $i=1,2, \ldots, M$ are indexes of design parameters against which the design alternatives are to be evaluated; $j=1,2, \ldots, J$ are indexes of design parameters to be evaluated; $k=1,2, \ldots, K$ are indexes of group of DMs involved into decision making process; $w_{i}^{k}$ are weighting coefficients representing relative importance of design parameters as evaluated by different DMs; $a_{i j}^{k}$ is evaluation score of $k$-th DM for performance of alternative $j$ against parameter $p_{i}$; coefficients $\alpha^{k}$ represent the weight of opinion of the $k$-th DM and $x_{j}$ are binary integer decision variables used to perform choice of a single alternative.

\section{Numerical testing}

In order to show the applicability of the proposed two-stage algorithm for WFLOP a number of numerical examples are tested and analysed.

\subsection{Input data for WFLOP}

This section provides the input data for numerical testing of the proposed algorithm in Section 2. A set of 30 different wind turbines are used to select the most appropriate type of turbine (Table 1).

Table 1. Wind turbines parameters

\begin{tabular}{|c|l|c|c|c|l|c|c|}
\hline$\#$ & Wind turbine type & $\begin{array}{c}\text { Rated } \\
\text { power, } \mathrm{kW}\end{array}$ & $\begin{array}{c}\text { Rotor } \\
\text { diameter, } \mathrm{m}\end{array}$ & $\#$ & Wind turbine type & $\begin{array}{c}\text { Rated } \\
\text { power, } \mathrm{kW}\end{array}$ & $\begin{array}{c}\text { Rotor } \\
\text { diameter, } \mathrm{m}\end{array}$ \\
\hline 1 & Enercon E-33 & 330 & 33.4 & 16 & SWT-2.3-113 & 2300 & 113.0 \\
\hline 2 & Enercon E-48 & 800 & 48.0 & 17 & SWT-2.3-108 & 2300 & 108.0 \\
\hline 3 & Enercon E-53 & 800 & 52.9 & 18 & SWT-2.3-93 & 2300 & 93.0 \\
\hline 4 & Vestas V52 & 850 & 52.0 & 19 & Enercon E-92 & 2350 & 92.0 \\
\hline 5 & Vestas V60 & 850 & 60.0 & 20 & C96 Clipper Windpower & 2500 & 96.0 \\
\hline 6 & Enercon E-44 & 900 & 44.0 & 21 & Vestas V100 & 2600 & 100.0 \\
\hline 7 & GAMESA AE-61-1320 & 1320 & 61.0 & 22 & Enercon E-82 & 3000 & 82.0 \\
\hline 8 & Vestas V82 & 1650 & 82.0 & 24 & Vestas V90 & 3000 & 90.0 \\
\hline 9 & M Torres & 1650 & 77.0 & 25 & Vestas V112 & 3000 & 112.0 \\
\hline 10 & Vestas V100 & 1800 & 100.0 & 23 & Enercon -101 & 3050 & 101.0 \\
\hline 11 & Vestas V80 & 2000 & 80.0 & 26 & SWT-3.6-120 & 3600 & 120.0 \\
\hline 12 & Enercon E-82 & 2000 & 82.0 & 27 & SWT-3.6-107 & 3600 & 107.0 \\
\hline 13 & Enercon E-70 & 2300 & 71.0 & 28 & SWT-6.0-154 & 6000 & 154.0 \\
\hline 14 & SWT-2.3-82 VS & 2300 & 82.4 & 29 & Vestas V164-7.0 & 7000 & 164.0 \\
\hline 15 & Enercon E2-82 & 2300 & 82.0 & 30 & Enercon E-126 & 7580 & 127.0 \\
\hline
\end{tabular}


The solution of the multi-objective problem formulated on Step 1.2 determines: Pareto-optimal wind turbines type and rotor diameter $D_{\mathrm{wt}}$; values of separation coefficients $k_{x}$ and $k_{y}$; number of turbines consistent with separation distances; placement of turbines within wind farm site in rows and columns taking into account wind direction. This solution defines wind farm Pareto-optimal layout. Three wind direction cases are tested: a) for uniform wind direction (Fig. 2a) with boundaries for separation coefficients $k_{x}^{\min }=k_{y}^{\min }=4.5, k_{x}^{\max }=k_{y}^{\max }=5.5$; b) for prevailing wind direction as in Fig. $2 \mathrm{~b}$ with $k_{x}^{\min }=1.5, k_{x}^{\max }=3, k_{y}^{\min }=8, k_{y}^{\max }=12$; for prevailing wind direction as in Fig. $2 \mathrm{c}$ with $k_{x}^{\min }=8, k_{x}^{\max }=12, k_{y}^{\min }=1.5$, $k_{y}^{\max }=3$. The wind farm annual energy production can be represented as AEP $=2628 N P_{\mathrm{wt}}$ using $h_{y}=8760$ hours over year and wind power utilization coefficient $\eta=0.3$. A rectangular wind farm with area of $4 \mathrm{~km}^{2}\left(L_{x}=4 \mathrm{~km}\right.$ and $\left.L_{y}=1 \mathrm{~km}\right)$ is considered with three different cases for wind directions as shown in Fig. 2.
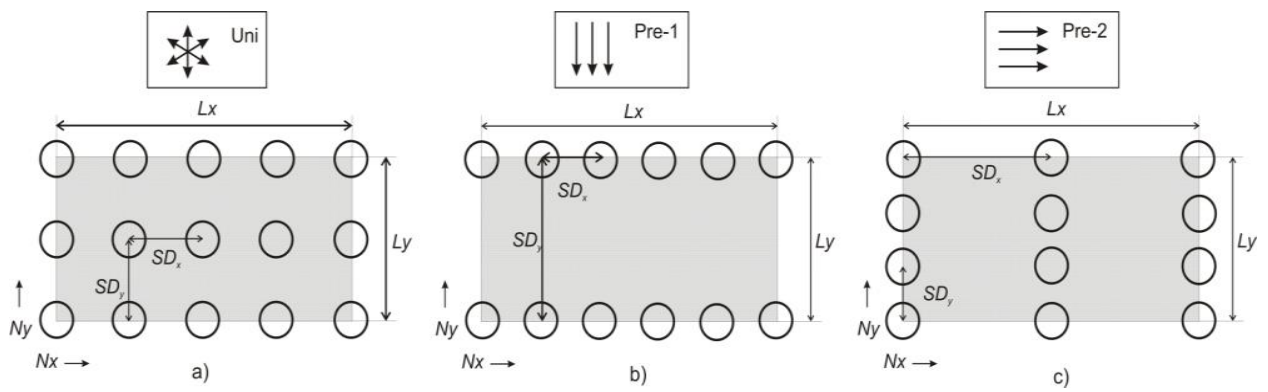

Fig. 2. Orientations of wind toward the shape of wind farm

\subsection{Generation of alternatives}

The formulated multi-objective optimization problem on Step 1.2 is solved by Weighted Sum (WS) and Lexicographic Method (LM). They are based on priori articulation of the DM preferences but the solution information by both methods can be used also with posterior preferences handling to generate different alternatives. These two methods are chosen due to their easy establishment of DM preferences toward the formulated objectives.

The weighted sum method is one of the most common multi-objective optimization methods based on scalarization techniques [9, 14]. It aggregates the different objectives as a weighted linear sum of their normalization. The DM preferences are expressed by imposing weight coefficients $w_{j}$ about the importance of different objectives. These weight coefficients are assigned to normalized form of objectives. The normalization scheme used here yields to non-dimensional objective function with values between 0 and 1 regardless of their original range [22]. The maximized objective for AEP and the minimized objective for costs are normalized by expressions:

$$
\begin{gathered}
f_{1}^{*}=\left(f_{\mathrm{AEP}}-f_{\mathrm{AEP}}^{\min }\right)\left(f_{\mathrm{AEP}}^{\max }-f_{\mathrm{AEP}}^{\min }\right)^{-1}, \\
f_{2}^{*}=\left(f_{\text {costs }}^{\max }-f_{\text {costs }}\right)\left(f_{\mathrm{costs}}^{\mathrm{max}}-f_{\mathrm{costs}}^{\min }\right)^{-1},
\end{gathered}
$$

where $f_{\mathrm{AEP}}^{\min }, f_{\mathrm{AEP}}^{\max }, f_{\text {costs }}^{\min }, f_{\text {costs }}^{\max }$ are the lowest and the greatest values of maximized 
objective for AEP and minimized objective for costs defined via solutions of the four single objective tasks:

- $\min \mathrm{AEP}=h_{y} \eta N P_{\mathrm{wt}}$ s.t. (2)-(11),

- $\max \mathrm{AEP}=h_{y} \eta N P_{\mathrm{wt}}$ s.t. (2)-(11),

- $\min \operatorname{Costs}=N\left(\frac{2}{3}+\frac{1}{3} e^{-0.00174 N^{2}}\right)$, s.t., (2)-(11),

- $\max$ Costs $=N\left(\frac{2}{3}+\frac{1}{3} e^{-0.00174 N^{2}}\right)$, s.t., (1)-(11),

The implementation of the WS method transforms the initial multi-objective problem (1)-(11) into single objective problem as

$$
\max \sum_{j=1}^{2} w_{j} f_{j}^{*}
$$

subject to constraints (2)-(11) plus additional constraints for the weighting coefficients:

$$
\begin{gathered}
\sum_{j=1}^{2} w_{j}=1, \\
w_{j}>0, \quad j=1,2 .
\end{gathered}
$$

The different wind farm design scenarios are simulated by using of 2 different

\begin{tabular}{|c|c|c|c|c|c|c|c|c|}
\hline \multirow[b]{2}{*}{ Task } & \multicolumn{2}{|c|}{ Input data } & \multicolumn{6}{|c|}{ Solution results } \\
\hline & $\begin{array}{c}\text { Wind } \\
\text { direction }\end{array}$ & \begin{tabular}{|c|}
$\begin{array}{c}\text { Weight } \\
\text { coefficients }\end{array}$ \\
\end{tabular} & $\begin{array}{c}\text { Turbine } \\
\text { type }\end{array}$ & $\begin{array}{c}\text { Turbines } \\
\text { number }\end{array}$ & $\begin{array}{c}\text { Separation } \\
\text { coefficients }\end{array}$ & \begin{tabular}{|c|} 
AEP, \\
MW.h per 1 year \\
\end{tabular} & Costs & Alternative \\
\hline WS-1 & \multirow{2}{*}{ Uniform } & $\begin{array}{l}w_{\mathrm{p}}=0.9 \\
w_{\mathrm{c}}=0.1\end{array}$ & \#13 & 52 & $k_{x}=4.69 ; k_{y}=4.69$ & 314,309 & 34.824 & A-1 (WS) \\
\hline WS-2 & & $\begin{array}{l}w_{\mathrm{p}}=0.1 \\
w_{\mathrm{c}}=0.9\end{array}$ & \#27 & 27 & $k_{x}=4.67 ; k_{y}=4.67$ & 255,442 & 20.531 & A-2 (WS) \\
\hline WS-3 & \multirow{2}{*}{$\begin{array}{c}\text { Predominant } \\
\text { on } Y \text { axis } \\
\text { (Pre-1) }\end{array}$} & $\begin{array}{l}w_{\mathrm{p}}=0.9 \\
w_{\mathrm{c}}=0.1\end{array}$ & \#27 & 50 & $k_{x}=1.56 ; k_{y}=9.35$ & 473,040 & 33.548 & A-3 (WS) \\
\hline WS-4 & & $\begin{array}{l}w_{\mathrm{p}}=0.1 \\
w_{\mathrm{c}}=0.9\end{array}$ & \#26 & 46 & $k_{x}=1.52 ; k_{y}=8.33$ & 435,197 & 31.053 & A-4 (WS) \\
\hline WS-5 & \multirow{2}{*}{$\begin{array}{c}\text { Predominant } \\
\text { on } X \text { axis } \\
(\text { Pre-2) }\end{array}$} & $\begin{array}{l}w_{\mathrm{p}}=0.9 \\
w_{\mathrm{c}}=0.1\end{array}$ & \#22 & 63 & $k_{x}=1.52 ; k_{y}=8.13$ & 496,692 & 42.021 & A-5 (WS) \\
\hline WS-6 & & $\begin{array}{l}w_{\mathrm{p}}=0.1 \\
w_{\mathrm{c}}=0.9\end{array}$ & \#30 & 24 & $k_{x}=1.57 ; k_{y}=10.50$ & 478,086 & 18.936 & A-6 (WS) \\
\hline
\end{tabular}
sets of weighting coefficients and three different wind direction cases. The solution results are shown in Table 2.

The WS method allows easy and intuitive definition of different preferences for the objectives. Simulation of different design scenarios can be done by posterior articulation of preference information, i.e., by altering the objectives' weights to yield different Pareto-optimal points.

Another way to handle multi-objective optimization problems by apriori articulation of the DM preferences is the lexicographic method. Using of this method requires ranking of objectives by the DM and optimization them in order one at a time. The general description of this method can be summarized as solution of sequence of single-objective optimization problems: subject to

$$
\min F_{j}(x), \quad x \in X, j=1,2, \ldots, k,
$$

$$
F_{j}\left(x_{j}\right) \leq \varepsilon_{j} F_{j}\left(x_{j}^{*}\right), \quad j=1,2, \ldots, i-1, i>1,
$$

where $j$ represents the objective function position in the sequence, $F_{j}\left(x_{j}^{*}\right)$ is the optimum of the $j$-th objective function found on the $j$-th iteration, and $\varepsilon_{j}$ is tolerance 
determined by the DM to expand the feasible region [23]. The solution of each single objective problem gives a limiting measure for that objective which is used to define a proper restriction on the next step when the next objective is optimized and so on. The Pareto-optimal solution is defined on the last step of the described optimization procedure. Two cases of lexicographic ordering are numerically tested.

Case 1. Wind farm AEP as the foremost objective and the optimization procedure for that case is as follow:

Step 1.1. Solving of the optimization task

$$
\max \mathrm{AEP}=h_{y} \eta N P_{\mathrm{wt}},
$$

subject to (2)-(11).

Step 1.2. Solving of the optimization task

$$
\min \text { Costs }=N\left(\frac{2}{3}+\frac{1}{3} e^{-0.00174 N^{2}}\right),
$$

subject to (2)-(11) and additional restriction regarding the energy output

$$
\mathrm{AEP}=h_{y} \eta N P_{\mathrm{wt}} \geq \varepsilon \mathrm{AEP}^{\max } .
$$

The coefficient $\varepsilon$ represents the degree of proximity to the optimal value of $\mathrm{AEP}=\left(h_{y} \eta N P_{\mathrm{wt}}\right)^{\max }$ calculated on the first step. Two values $\varepsilon=0.7$ and $\varepsilon=0.9$ are used to define two different design alternatives.

Ca se 2. Costs are considered as more important than AEP. The corresponding optimization procedure is as follow:

Step 2.1. Solving of the optimization task

$$
\min \text { Costs }=N\left(\frac{2}{3}+\frac{1}{3} e^{-0.00174 N^{2}}\right),
$$

subject to (2)-(11).

Step 2.2. Solving of the optimization task

$$
\max \mathrm{AEP}=h_{y} \eta N P_{\mathrm{wt}} \text {, }
$$

subject to (2)-(11) and additional restriction regarding the Costs

$$
\text { Costs }=N\left(\frac{2}{3}+\frac{1}{3} e^{-0.00174 N^{2}}\right) \leq \varepsilon \operatorname{Costs}^{\min },
$$

\begin{tabular}{|c|c|c|c|c|c|c|c|c|}
\hline \multirow[b]{2}{*}{ Task } & \multicolumn{2}{|l|}{ Input data } & \multicolumn{6}{|c|}{ Solution results } \\
\hline & Wind Direction & $\varepsilon$ & $\begin{array}{c}\text { Turbine } \\
\text { type }\end{array}$ & $\begin{array}{l}\text { Turbines } \\
\text { number }\end{array}$ & $\begin{array}{c}\text { Separation } \\
\text { coefficients }\end{array}$ & AEP, MW.h per 1 year & Costs & Alternative \\
\hline \multicolumn{9}{|c|}{ a) lexicographic ordering $\rightarrow$ AEP, Costs } \\
\hline Lp-1 & \multirow{2}{*}{ Uniform } & 0.7 & \# 27 & 27 & $k_{x}=4.67 ; k_{y}=4.67$ & 255,442 & 20.531 & A-1 \\
\hline Lp-2 & & 0.9 & $\# 13$ & 52 & $k_{x}=4.69 ; k_{y}=4.69$ & 314,309 & 34.823 & A-2 \\
\hline Lp-3 & \multirow{2}{*}{$\begin{array}{c}\text { Predominant } \\
\text { on } Y \text { axis (Pre-1) }\end{array}$} & 0.7 & \#26 & 36 & $k_{x}=1.96 ; k_{y}=8.33$ & 340,589 & 25.253 & A-3 \\
\hline Lp-4 & & 0.9 & $\# 26$ & 46 & $k_{x}=1.52 ; k_{y}=8.33$ & 435,197 & 31.053 & A-4 \\
\hline Lp-5 & \multirow{2}{*}{$\begin{array}{c}\text { Predominant } \\
\text { on } X \text { axis (Pre-2) }\end{array}$} & 0.7 & $\# 30$ & 20 & $k_{x}=1.97 ; k_{y}=10.50$ & 398,405 & 16.657 & A-5 \\
\hline Lp-6 & & 0.9 & $\# 30$ & 24 & $k_{x}=1.58 ; k_{y}=10.45$ & 478,086 & 18.936 & A-6 \\
\hline \multicolumn{9}{|c|}{ b) lexicographic ordering $\rightarrow$ Costs, AEP } \\
\hline Lc-1 & \multirow{2}{*}{ Uniform } & 1.3 & $\# 27$ & 27 & $k_{x}=4.67 ; k_{y}=4.67$ & 255,442 & 20.531 & $\overline{\mathrm{A}-1}$ \\
\hline Lc-2 & & 1.1 & \# 27 & 27 & $k_{x}=4.67 ; k_{y}=4.67$ & 255,442 & 20.531 & A-2 \\
\hline Lc-3 & \multirow{2}{*}{$\begin{array}{c}\text { Predominant } \\
\text { on } Y \text { axis (Pre-1) }\end{array}$} & 1.3 & $\# 27$ & 36 & $k_{x}=2.20 ; k_{y}=9.35$ & 340,589 & 25.258 & A-3 \\
\hline Lc-4 & & \begin{tabular}{|l|}
1.1 \\
\end{tabular} & $\# 27$ & 28 & $k_{x}=2.87 ; k_{y}=9.35$ & 264,902 & 21.052 & A-4 \\
\hline Lc-5 & \multirow{2}{*}{$\begin{array}{c}\text { Predominant } \\
\text { on } X \text { axis (Pre- } 2)\end{array}$} & 1.3 & $\# 30$ & 24 & $k_{x}=10.50 ; k_{y}=1.57$ & 478,086 & 18.936 & A-5 \\
\hline Lc-6 & & \begin{tabular}{|l|}
1.1 \\
\end{tabular} & $\# 30$ & 16 & $k_{x}=10.50 ; k_{y}=2.62$ & 318,724 & 14.083 & A-6 \\
\hline
\end{tabular}

where the coefficient $\varepsilon$ represents the degree of proximity of the costs to the optimal value $N\left(\frac{2}{3}+\frac{1}{3} e^{-0.00174 N^{2}}\right)^{\mathrm{min}}$ calculated on the first step. The values of $\varepsilon$ used for simulation of different design scenarios are $\varepsilon=1.3$ and $\varepsilon=1.1$.

The results of the lexicographic method implementation are shown in Table 3.

Table 3. Design alternatives by lexicographic method solutions 


\subsection{Filtering of identical alternatives}

Comparison of design alternatives from Table 2 and Table 3 shows that there are identical alternatives. For example, in case of uniform wind the identical alternatives are A-1(WS) and A-2(Lp), A-2(WS), A-1(Lp), A-1(Lc) and A-2(Lc). For predominant wind (case Pre-1) the identical alternatives are A-3(Lp) and A-3(Lc), A-4(WS) and A-4(Lp) and for opposite direction (case Pre-2) the identical alternatives are A-6(WS), A-6(Lp) and A-5(Lc). The identical design alternatives are considered as one unique alternative as shown in Table 4.

Table 4. Unique design alternatives as result of execution of Stage 1
\begin{tabular}{|c|c|c|c|c|c|c|}
\hline Unique alternatives & Turbine type (from Table 1) & Turbines number & $\mathrm{SD}_{x}, \mathrm{~m}$ & $\mathrm{SD}_{y}, \mathrm{~m}$ & $\begin{array}{c}\text { AEP, } \\
\text { MW.h per 1 year }\end{array}$ & Costs \\
\hline \multicolumn{7}{|c|}{ Uniform wind direction } \\
\hline A-1 (uni) & $\# 13$ & 52 & 333.4 & 333.4 & 314,309 & 34.824 \\
\hline A-2 (uni) & $\# 27$ & 27 & 500.0 & 500.0 & 255442 & 20.531 \\
\hline \multicolumn{7}{|c|}{ Predominant wind direction (Pre-1) } \\
\hline A-1 (Pre-1) & $\# 27$ & 50 & 166.7 & 1000.0 & 473,040 & 33.55 \\
\hline A-2 (Pre-1) & $\# 26$ & 46 & 181.8 & 1000.0 & 435,197 & 31.05 \\
\hline A-3 (Pre-1) & $\# 26$ & 36 & 235.3 & 1000.0 & 340,589 & 25.25 \\
\hline A-4 (Pre-1) & $\# 27$ & 28 & 307.7 & 1000.0 & 264,902 & 21.05 \\
\hline \multicolumn{7}{|c|}{ Predominant wind direction (Pre-2) } \\
\hline A-1 (Pre-2) & $\# 22$ & 63 & 666.7 & 125.0 & 496,692 & 42.02 \\
\hline A-2 (Pre-2) & $\# 30$ & 24 & 1333.4 & 200.0 & 478,086 & 18.94 \\
\hline A-3 (Pre-2) & $\# 30$ & 20 & 1333.4 & 250.0 & 398,405 & 16.66 \\
\hline A-4 (Pre-2) & $\# 30$ & 16 & 1333.4 & 333.4 & 318,724 & 14.08 \\
\hline
\end{tabular}

The identical alternatives for different wind directions are visualized as overlapping circles (Fig. 3).
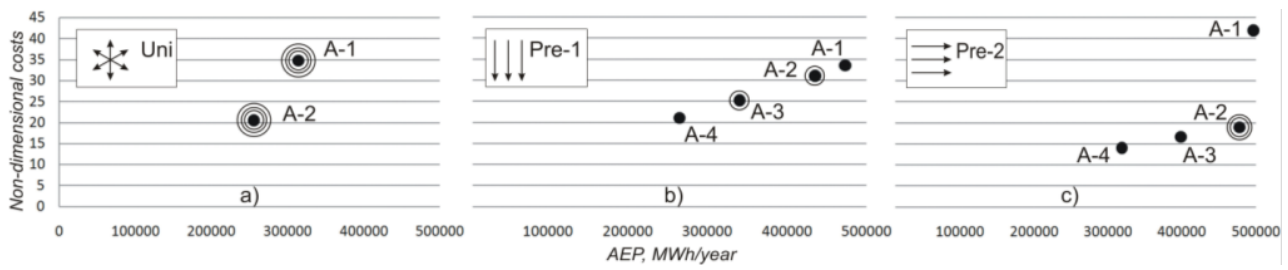

Fig. 3. AEP-Costs diagram for different design alternatives: for uniform wind direction (a); for two cases (Pre-1 and Pre-2) of predominant wind direction (b) and (c)

After filtering not identical alternatives of Table 4 are stored for use on the Step 2.3 of the algorithm.

\subsection{Determination of optimal alternative by group decision making}

Three wind farm design parameters shown in Table 5 are used and evaluated by group of three DMs. The WDM representing WFLOP via GDM considers the case Pre-2 data which is characterised by four unique design alternatives (Table 5). Three different experts - engineer (DM-1), a financial expert (DM-2) and ecologist (DM3) are involved in the process of alternatives evaluation toward three wind farm 
design parameters (energy output, costs and number of turbines). For each of these experts a corresponding weighting coefficient is assigned to represent its opinion importance.

Table 5. Input WDM data for testing of GDM

\begin{tabular}{|c|c|c|c|c|c|c|c|c|c|c|c|c|c|c|c|}
\hline \multirow{2}{*}{$\begin{array}{c}\text { Design } \\
\text { parameters }\end{array}$} & \multicolumn{3}{|c|}{$\begin{array}{c}\text { Parameters' } \\
\text { weights }\end{array}$} & \multicolumn{3}{|c|}{$\begin{array}{c}\text { Alternative A-1 } \\
\text { evaluations }\end{array}$} & \multicolumn{3}{|c|}{$\begin{array}{c}\text { Alternative A-2 } \\
\text { evaluations }\end{array}$} & \multicolumn{3}{|c|}{$\begin{array}{c}\text { Alternative A-3 } \\
\text { evaluations }\end{array}$} & \multicolumn{3}{|c|}{$\begin{array}{c}\text { Alternative A-4 } \\
\text { evaluations }\end{array}$} \\
\hline & DM-1 & DM-2 & DM-3 & DM-1 & DM-2 & DM-3 & DM-1 & DM-2 & DM-3 & DM-1 & DM-2 & DM-3 & DM-1 & DM-2 & DM-3 \\
\hline AEP MW.h per 1 year & 10 & 4 & 3 & 10 & 8 & 4 & 8 & 6 & 7 & 6 & 5 & 6 & 4 & 3 & 5 \\
\hline Costs & 4 & 10 & 3 & 5 & 2 & 4 & 6 & 7 & 5 & 7 & 6 & 6 & 8 & 5 & 7 \\
\hline Turbines nt & 5 & 8 & 10 & 3 & 2 & 2 & 4 & 6 & 5 & 5 & 7 & 7 & 6 & 6 & 8 \\
\hline
\end{tabular}

The solutions results based on solution of single-objective integer optimization task (12)-(15) for 4 sets of values of DMs' weights $\alpha$ are shown in Table 6.

Table 6. GDM results for different weights of DMs
\begin{tabular}{|r|c|c|c|c|}
\hline \multirow{2}{*}{ Tasks } & Weighting coefficients of DMs & Best alternative \\
\cline { 2 - 5 } & $\alpha_{1}$ & $\alpha_{2}$ & $\alpha_{3}$ & \\
\hline GDM-1 & 0.5 & 0.5 & 0.5 & A-3 \\
\hline GDM-2 & 0.9 & 0.2 & 0.2 & A-2 \\
\hline GDM-3 & 0.2 & 0.9 & 0.2 & A-2 \\
\hline GDM-4 & 0.2 & 0.2 & 0.9 & A-4 \\
\hline
\end{tabular}

The graphical illustration of the optimal layout design alternatives obtained as result of execution of Stage 2 are shown in Fig.4.

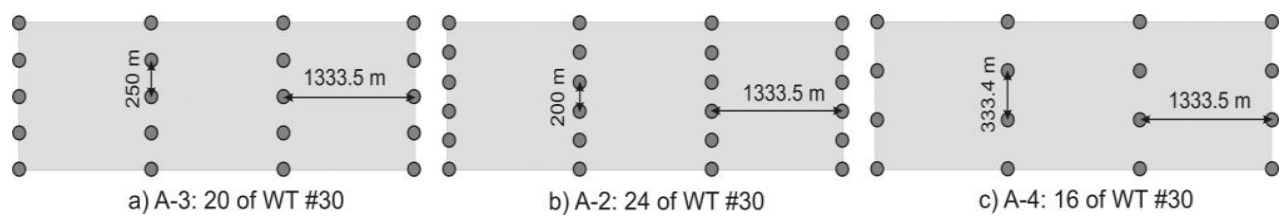

Fig. 4. Graphical representation of: Alternative-3 (a); Alternative-2 (b); Alternative-4 (c)

\section{Results analysis and discussions}

The formulated optimizations tasks are solved by Lingo V. 12 system (http://www.lindo.com). The solution algorithm is branch-and-bound and solution times for the described numerical examples are about $1 \mathrm{~s}$. Lingo' status windows for some examples of the formulated discrete combinatorial optimization tasks are shown in Fig. 5.

The nonlinearity of the defined optimization tasks leads to local optimums. This nonlinearity is caused from using of widely accepted by many researches nonlinear formulation of costs $[15,25,36]$. If linear discrete formulation of costs is used the solutions of the formulated in the article tasks will define global optimums.

The used two solution methods - weighted sum and lexicographic method show the benefits of using multi-objective combinatorial optimization of as an analytical simulation tool for determination of different design alternatives. 


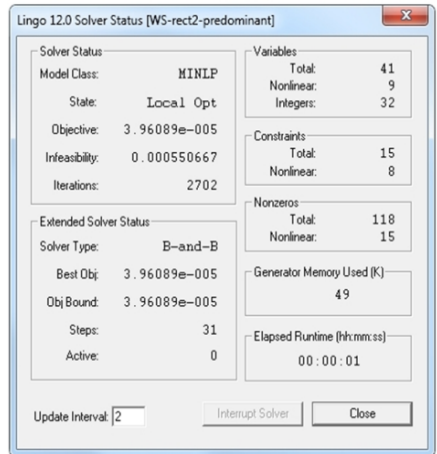

(a)

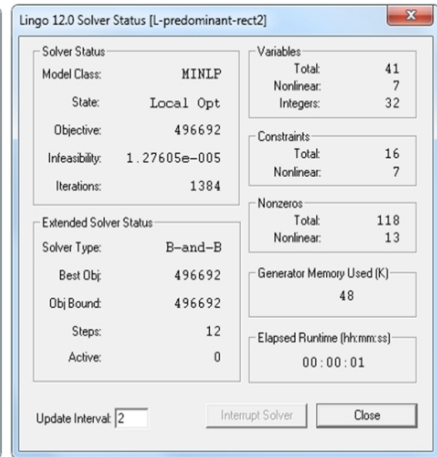

(b)

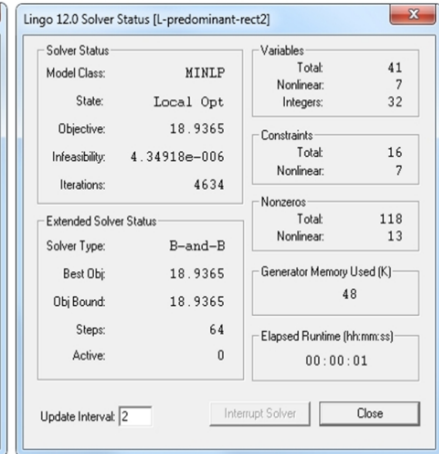

(c)

Fig. 5. Lingo status window for: task WS-5 (a); two stages of task Lp-6 (b) and (c)

The results of solution by weighted sum method show that stronger preference on AEP leads to the choice of turbines with a smaller rotor diameter. As the rotor diameter is decisive for considering of WE by proper separation distances, the smaller rotor diameter leads to increasing of turbines number and as a consequence - to increasing of the energy yield. The opposite preference (Costs over AEP) results in choice of more powerful turbines with a bigger rotor diameter. This leads to reducing of the turbines number that in turn decreases the costs compared to the first case. The site orientation towards the prevailing wind direction also affects the Pareto-optimal design alternatives.

The lexicographic ordering of the objectives is another easy implementation method to simulate different scenarios for WFLOP. Variety of Pareto-optimal solutions (design alternatives) can be determined by different lexicographic ordering of the objectives and by different values of coefficient $\varepsilon$. For lexicographic ordering AEP, Costs, when $\varepsilon$ changes from 0.7 up to 0.9 , the turbines' number increases and the AEP is approaching closer to its maximum. In lexicographic ordering Costs before AEP and value of $\varepsilon$ decreases from 1.3 to 1.1, the result leads to decreasing of the turbines' number and Costs are getting closer to their minimum. Changing the value of $\varepsilon$ coefficient allows flexible adjustment of DM preferences in terms of tradeoffs between objectives, i.e., provides various design alternatives.

Numerical testing demonstrates that both methods are adequate for the generating of wind farm layout design alternatives. The weighted sum method has limitations in regard to representation of compromises that DM is willing to make among objectives and also requires normalization of the objectives. The lexicographic method allows a more precise tuning of these trade-offs by considering the desirable degree of proximity to the "best" values of objectives. The discrete nature of the turbines' type choice defines in some cases overlapping Pareto-optimal alternatives. This overlapping reduces the number of design alternatives to be evaluated that helps for facilitating the selection of the final alternative.

In contrast to other methods for GDM, the proposed GDM approach considers simultaneously not only different DMs weights about design parameters (criteria) and alternatives evaluations but also group members' opinions weights. Apparently, the assignment of weights to the group members' opinions influences on decision process 
and selection of collective alternative. That is why Alternative-2 (A-2) (Table 4, case Pre-2) appears to be the best for engineers and economists while Alternative-3 (A-3) is accepted as best alternative if the weights of experts are equal. Unlike other GDM approaches the described approach provides globally optimal alternative as a solution of the formulated single-objective integer linear optimization task.

From engineering point of view, it is important to get to the problems solution by the most direct and effective way. This requires interdisciplinary approach to modelling of the decision-making processes and using of quantitative optimization methods. The proposed in the article algorithm combines two approaches generating of multiple alternative design solutions for WFLOP and choice of the best alternative by group decision making. This idea expresses the complexity and multidimensionality of the WFLOP. In contrast to mass used genetic and other heuristic algorithms, the described algorithm is based on combinatorial optimization modelling. This approach allows using of the proven over the years computational efficiency of the algorithms for linear, nonlinear and integer optimization. The used modelling approach on Stage 1 of the algorithm defines wind turbines of the same type. This is preferred case for commercial wind farms because it reduces installation costs, maintenance cost, etc., [4]. Like many other published results for WFLOP $[10,19,26,38]$, the testing of the proposed algorithm is done for a rectangular wind farm site. The rectangular shape could be quite practical for offshore wind farms and for onshore wind farms located on flat terrain. The placement of turbines in rows and columns has the advantage that small deviations of prevailing wind direction lead to increasing of the separation distances between turbines, i.e., to decreasing the influence of WE (Fig. 6).

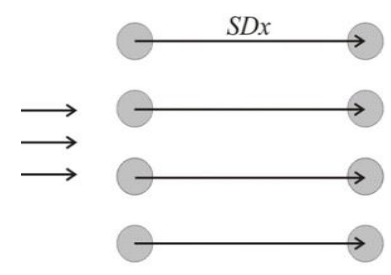

a)

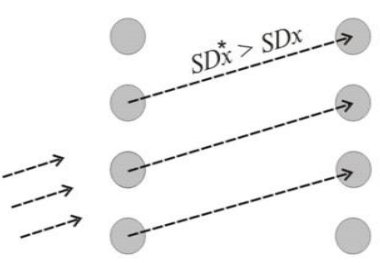

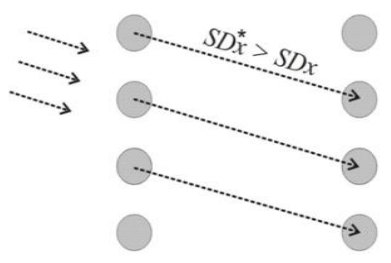

Fig. 6. Separation distances between turbines: predominant wind direction $\left(\mathrm{SD}_{x}\right)(\mathrm{a})$; small deviations of predominant wind direction $\left(\mathrm{SD}_{x}^{*}\right)(\mathrm{b})$

To justify the effectiveness and applicability of the proposed algorithm it is compared with other WFLOP algorithms. The main idea of the proposed algorithm - combining the determination of multiple design alternatives for WFLOP and using of GDM for choice of the best alternative, cannot be compared because it has no analogue in other publications on WFLOP. The other feature of the algorithm, namely using of discrete combinatorial optimization modelling, can be compared with other modelling approaches. Widely used for WFLOP are GA and other metaheuristic algorithms. Usually that kind of algorithms are used for problems that cannot be easily solved and they give nearly the right answer or provide a solution not for all instances of the problem [18]. The widely used GA have some known drawbacks: A solution is "better" only in comparison to other, presently known solutions; in reality 
they have no concept of "optimal solution" or way to test whether a solution is optimal; stop criterion is not always clear in every problem, aside from the runtime length, or the number of iterations or candidate solutions to explore (http://www.solver.com/genetic-evolutionary-introduction). Sometimes it is possible GA to converge not only to local optima but even to arbitrary points. The GA repeated fitness function evaluation requires essential computation power and is time consuming.

5.1. Comparison of computing performance of proposed algorithm and other algorithms for WFLOP

To compare the computing performance of the proposed two-stage algorithm with other algorithms for WFLOP the task WS-6 is modified and solved for different wind farm areas as shown in Table 7.

Table 7. Comparison of the proposed algorithm with other algorithms

\begin{tabular}{|c|c|c|c|c|}
\hline Algorithm & Turbines number & Area & Runtime & Authors \\
\hline Random search & 80 (fixed) & $4 \times 5 \mathrm{~km}$ & $\begin{array}{l}3170.21-3498.25 \mathrm{~s} \\
\text { (per run) }\end{array}$ & [11] \\
\hline Genetic & 15 & $2 \times 2 \mathrm{~km}$ & $\begin{array}{c}16000 \mathrm{~s} \\
\text { (on CRAY XMP14) }\end{array}$ & [26] \\
\hline MIP model & limited to $<10$ & & $<1 \mathrm{~h}$ & [5] \\
\hline \begin{tabular}{|c|} 
Evolutionary NSGA-II, \\
SPEA2, IBEA \\
\end{tabular} & $30,50,70$ (fixed) & $3 \times 3 \mathrm{~km}$ & $<15 \min$ & [39] \\
\hline $\begin{array}{l}\text { Stochastic evolutionary } \\
\text { algorithm (NSGA-II) }\end{array}$ & $5,10,15$ (fixed) & $3 \times 3 \mathrm{~km}$ & $\begin{array}{c}19.75 \mathrm{~h} ; 70.87 \mathrm{~h} ; \\
149.75 \mathrm{~h}\end{array}$ & [38] \\
\hline Evolutionary algorithm & $4,5,20$ (fixed) limited to $<50$ & $20 \times 20$ grid & $90 \mathrm{~min}$ & [25] \\
\hline MIP & $10-100$ (fixed) & $1.7 \times 2 \mathrm{~km}$ & limited to $1800 \mathrm{~s}$ & [6] \\
\hline MIP & $40,50,60,70$ (fixed) & $4 \times 4 \mathrm{~km}$ & $\begin{array}{c}\text { within wall-clock } \\
\text { time of } 200 \mathrm{~s}\end{array}$ & [19] \\
\hline MINLP (Task WS-6) & defined as 63 of Enercon E-82 & $1 \times 4 \mathrm{~km}$ & about $1 \mathrm{~s}$ & present work \\
\hline MINLP (Task WS-6) & defined as 18 of SWT-3.6-120 & $1.7 \times 2 \mathrm{~km}$ & about $1 \mathrm{~s}$ & present work \\
\hline MINLP (Task WS-6) & defined as 21 of SWT-3.6-120 & $2 \times 2 \mathrm{~km}$ & about $1 \mathrm{~s}$ & present work \\
\hline MINLP (Task WS-6) & defined as 48 of Enercon E-126 & $3 \times 3 \mathrm{~km}$ & about $1 \mathrm{~s}$ & present work \\
\hline MINLP (Task WS-6) & defined as 84 of Enercon E-126 & $4 \times 4 \mathrm{~km}$ & about $1 \mathrm{~s}$ & present work \\
\hline MINLP (Task WS-6) & defined as 108 of Enercon E-126 & $4 \times 5 \mathrm{~km}$ & about $1 \mathrm{~s}$ & present work \\
\hline MINLP (Task WS-6) & defined as 540 of Enercon E-126 & $20 \times 5 \mathrm{~km}$ & about $1 \mathrm{~s}$ & present work \\
\hline MINLP (Task LP-6) & defined as 24 Enercon E-126 & $1 \times 4 \mathrm{~km}$ & $\begin{array}{c}\text { about } 2 \mathrm{~s} \\
(2 \text { runs of } 1 \mathrm{~s})\end{array}$ & presen \\
\hline
\end{tabular}

A testing experiment is done with increased wind farm area to $100 \mathrm{~km}^{2}$ to show the computational time of the task and as it is seen from Fig. 7, the time remain in the range of one second.

Some remarks can be stated in regard to the application of two-stage algorithm for WFLOP and usage of mathematical model (1)-(11):

- Most of the WFLOP results are related with preliminary fixed number of turbines $[5,10,11,19,28,36,39]$. It is not clear how this number is determined and if it is optimal for the considered wind site area. In contrast, the proposed algorithm allows determination of Pareto-optimal number and type of turbines for the given wind farm area while considering the wind direction and recommendations for separations distances to avoid the negative influence of the WE. 
- An advantage of the described combinatorial modelling approach is that the number of variables of the defined optimization problems depends mainly on the number of turbines, from which selection is made. The area of the wind farm does not affect the dimension of the tasks (Fig. 5 and Fig. 7).

- Both stages of the proposed algorithm can be used separately. The generated on Stage 1 set of design alternatives could be evaluated by other GDM approaches. On the other hand, if variety alternatives are known or given in advance the described in the Stage 2 approach for GDM would be quite effective for choice of best alternative.

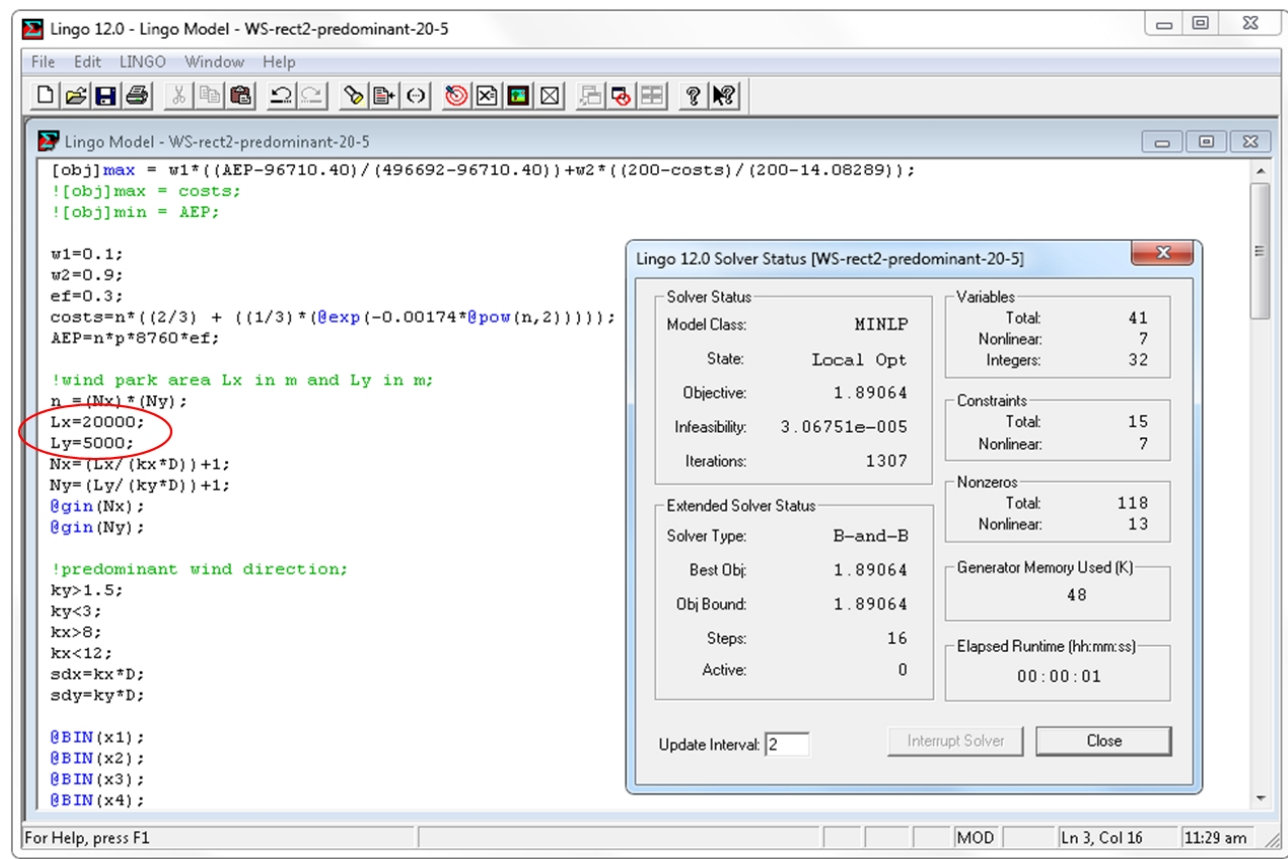

Fig. 7. Lingo status windows of task WS- 6 for site dimensions $20 \times 5 \mathrm{~km}$

\section{Conclusion}

In this article a two-stage placement algorithm with multi-objective optimization and group decision making is proposed. The main idea is to generate multiple Paretooptimal placement alternatives and to select one of them via group of experts to get the most suitable business decision. Using of multi-objective discrete combinatorial optimization, on the first stage of the algorithm provides different Pareto-optimal alternatives and can be consider as a flexible analytical tool to simulate different design scenarios. The different DMs preferences toward importance of used criteria simulate different design alternatives. All of these alternatives are used on the second stage of the proposed algorithm to select the most suitable alternative by group decision making. 
The described two-stage algorithm is numerical tested for design of wind farm layout to show its practical applicability. The runtimes for tested examples are in the range of a few seconds versus hundreds or thousands of seconds needed for the execution of other published algorithms. This demonstrates the effectiveness of the use of combinatorial optimization modelling for wind farm layout optimization problems.

The proposed two-stage algorithm can be applied for design of other types of engineering systems provided that their specificity is taken into account in modelling and formulation of corresponding optimization tasks.

\section{References}

1 B orissova, D., I. Mustakerov. Wind Power Plant Layout Design and Assessment Considering Forbidden Zones for Location of Turbines. - Adv. Modeling and Optimization, Vol. 19, 2017, pp. 29-38.

2 B or i s s o va, D., I. M u s t a k e r o v, D. K or s e mo v. Business Intelligence System via Group Decision Making. - Cybernetics and Information Technologies, Vol. 16, 2016, No 3, pp. 219-229.

3 B oris s ova, D., I. Mu stakerov. A Generalized Combinatorial Optimization Approach to Wind Power Plant Design. - Cybernetics and Information Technologies, Vol. 10, 2010, No 4, pp. 62-74.

4 Ch e n, Y., H. Li, B. H e, P. W a n g, K. J i n. Multi-Objective Genetic Algorithm Based Innovative Wind Farm Layout Optimization Method. - Energ. Convers. Manage., Vol. 105, 2015, pp. 1318-1327.

5 D o n ovan, S. Wind Farm Optimization. - In Proc. of Annual Conference of the Operations Research Society, Wellington, New Zealand, 2005.

6 D o n o v a n, S., G. N a t e s, H. W a t e r e r, R. A r c h e r. Mixed Integer Programming Models for Wind Farm Design. - In: Workshop on Mixed Integer Programming, Columbia University, New York City, 2008.

7 Du Pont, B., J. Cagan. An Extended Pattern Search Approach to Wind Farm Layout Optimization. - J. Mech. Design., Vol. 134, 2012.

8 Ehrgott, M. A Discussion of Scalarization Techniques for Multiple Objective Integer Programming. - Ann. Oper. Res., Vol. 147, 2006, pp. 343-360.

9 E i chfeld e r, G. Adaptive Scalarization Methods in Multiobjective Optimization. - Springer, Berlin Haidelberg, 2008.

10 Feng, J., W. Z. Shen. Modelling Wind for Wind Farm Layout Optimization Using Joint Distribution of Wind Speed and Wind Direction. - Energies, Vol. 8, 2015, pp. 3075-3092.

11 Feng, J., W. Z. S h e n. Solving the Wind Farm Layout Optimization Problem Using Random Search Algorithm. - Renew. Energ., Vol. 78, 2015, pp. 182-192.

12 F u 1 o p, J. Introduction to Decision Making Methods. Working Paper 05-6, 2005.

13 Gartner Research Methodologies. http://www.gartner.com/technology/research/methodologies

14 Genova, K., L. Kirilov, V. Guljash ki. New Reference-Neighbourhood Scalarization Problem for Multiobjective Integer Programming. - Cybernetics and Information Technologies, Vol. 13, 2013, No 1, pp. 104-114.

15 Grady, S. A., M. Y. Hus saini, M. M. Abdullah. Placement of Wind Turbines Using Genetic Algorithms. - Renew. Energ., Vol. 30, 2005, pp. 259-270.

16 S. Greco, M. Ehrgott, J. R. Figueira, Eds. Multiple Criteria Decision Snalysis: State of the Art Surveys. New York, Springer Verlag, 2016.

17 K a t i c, I., J. Hoj s tru p, N. O. J e n s e n. A Simple Model for Cluster Efficiency. - In: Proc. of European Wind Energy Association Conference and Exhibition, 1986, pp. 407-410.

$18 \mathrm{~K}$ o k a s h, N. An Introduction to Heuristic Algorithms. 2005. 
19 K u o, J. Y. J., D. A. R o me r o, C. H. A mo n. A Mechanistic Semi-Empirical Wake Interaction Model for Wind Farm Layout Optimization. - Enegry, Vol. 93, 2015, pp. 2157-2165.

$20 \mathrm{~K}$ w o n g, W.Y., P.Y.Z h a g, D. Ro m e ro, J. Mor a n, M. Morge n rot h, C. A m o n. MultiObjective Wind Farm Layout Optimization Considering Energy Generation and Noise Propagation with NSGA-II. - J. Mech. Design., Vol. 136, 2014.

21 L a r s o n, D.,V. C h a n g. A Review and Future Direction of Agile, Business Intelligence, Analytics and Data Science. - Int. J. Information Management Vol. 36, 2016, pp. 700-710.

$22 \mathrm{M}$ a r l e r, R.T., J.S. A r o r a. Function-Transformation Methods for Multi-Objective Optimization. - Eng. Optimiz., Vol. 37, 2005, pp. 551-570.

23 M a r l e r, R.T., J.S. A r o r a. Survey of Multi-Objective Optimization Methods for Engineering. Struct. Multidisc. Optim., Vol. 26, 2004, pp. 369-395.

$24 \mathrm{M}$ ar midis, G., S. L a z a r o u, E. P y r g i o t i. Optimal Placement of Wind Turbines in a Wind Park Using Monte Carlo Simulation. - Renew. Energ., Vol. 7, 2008, pp. 1455-1460.

25 M or a, J.C., J.M.C. B ar o n, J.M.R. S a n to s, M.B. P a y a n. An Evolutive Algorithm for Wind Farm Optimal Design. - Neurocomputing, Vol. 70, 2007, pp. 2651-2658.

26 Mos e t t i, G., C. Polon i, B. D i vi a c c o. Optimization of Wind Turbine Positioning in Large Windfarms by Means of a Genetic Algorithm. - J. Wind. Engand. Ind. Aerod., Vol. 51, 1994, pp. 105-116.

27 Mustakerov, I, D. Borissova. Wind Turbines Type and Number Choice Using Combinatorial Optimization. - Renew. Energ., Vol. 35, 2010, pp. 1887-1894.

28 O s t e r g a a r d, P. A. Reviewing Optimisation Criteria for Energy Systems Analyses of Renewable Energy Integration. - Energy, Vol. 4, 2009, pp. 1236-1245.

29 P e n e v a, V., I. P o p c h e v, Fuzzy Multi-Criteria Decision Making Algorithms. - Compt. Rend. Acad. bulg. Sci., Vol. 63, 2010, pp. 979-992.

30 P e n e v a, V., I. P o p c h e v. Models for Decision Making by Fuzzy Relations and Fuzzy Numbers for Criteria Evaluations. - Compt. Rend. Acad. bulg. Sci., Vol. 62, 2009, pp. 1217-1222.

31 P e n e va, V., I. P o p che v. Models for Fuzzy Multicriteria Decision Making Based on Fuzzy Relations. - Compt. Rend. Acad. bulg. Sci., Vol. 62, 2009, pp. 551-558.

32 Peneva, V., I. Popchev. Multicriteria Decision Making Based on Fuzzy Relations. Cybernetics and Information Technologies, Vol. 8, 2008, pp. 3-12.

33 P e n e va, V., I. P o p che v. Multicriteria Decision Making by Fuzzy Relations and Weighting Functions for the Criteria. - Cybernetics and Information Technologies, Vol. 9, 2009, pp. 58-71.

34 Perez, B., R. Minguez, R. Guanch e. Offshore Wind Farm Layout Optimization Using Mathematical Programming Techniques. - Renew. Energ., Vol. 53, 2013, pp. 389-399.

$35 \mathrm{~S}$ a a vedra-Moreno, B., S. S a lcedo-S anz, A. Paniagua-Tineo, L. Prieto, A. P ortill a-Figueras. Seeding Evolutionary Algorithms with Heuristics for Optimal Wind Turbines Positioning in Wind Farms. - Renew. Energ., Vol. 36, 2011, pp. 2838-2844.

36 S h a k o o r, R., M. Y. H a s s a n, A. R a h e e m, Y. K. W u. Wake Effect Modeling: A Review of Wind Farm Layout Optimization Using Jensen's Model. - Renew. Sust. Energ. Rev., Vol. 58, 2016, pp. 1048-1059.

37 S mith, G., W. S chlez, A. Liddell, A. Ne ubert, A. Pen a. Advanced Wake Model for Very Closely Spaced Turbines. - In: Proc. EWEC 2006, Athens. 2006.

38 S orkhabi, S. Y. D., D. A. Romero, G. K. Y a n, M. D. Gu, J. Mor a n, M. Morgenroth, C. H. A mo n. The Impact of Land Use Constraints in Multi-Objective Energy-Noise Wind Farm Layout Optimization. - Renew. Energ., Vol. 85, 2016, pp. 359-370.

39 T r a n, R., J. W u, C. D e n i s o n, T. A c k l i n g, M. W a g n e r, F. N e u m a n n. Fast and Effective Multi-Objective Optimisation of Wind Turbine Placement. - In: Proc. Genetic and Evolutionary Computation, 2013, pp. 1381-1388.

40 W a n, C., J. W a n g, G. Y a n g, X. Li, X. Z h a n g. Optimal Micro-Siting of Wind Turbines by Genetic Algorithms Based on Improved Wind and Turbine Models. - In: Proc. of 48th IEEE Conf. on Decision \& Control and the 28th Chinese Control Conference, 2009. 